\title{
Времяпролетные
}

\section{хромато-масс-спектрометры: особенности конструкции, возможности и преимущества}

М. Мисселвитц, Chemistry Matters, Bellefonte, Пенсильвания, США mmisselwitz192@gmail.com
УДК 543.07

Статья получена 15.01.2020

Принята к публикации 10.02.2020

\begin{abstract}
Приобретение масс-спектрометра (MC) - важная инвестиция в современную аналитическую лабораторию. При рассмотрении инструментальных платформ выбор хорошего масс-спектрометра для лаборатории расширяет ее функциональные границы, помогает в достижении целей, получении достоверных результатов, увеличении аналитических возможностей и экономии бюджета. Времяпролетный масс-спектрометр (ВПМС), характеризующийся высокой скоростью регистрации масс-спектров, в сочетании с газовой хроматографией (ГХ) может оказаться очень полезным в работе как научно-исследовательской, так и высокопроизводительной лаборатории, работающей по стандартным процедурам. ГХ-ВПМС - правильный выбор для обнаружения новых неизвестных соединений, одновременного количественного определения целевых аналитов и нецелевого анализа сложных образцов, увеличивающий производительность лаборатории благодаря возможности проведения высокоскоростной хроматографии. Области применения самые разнообразные, например, контроль загрязнения объектов окружающей среды, безопасность продуктов питания, управление отходами, анализ образцов растительных, животных тканей и биологических жидкостей в метаболомике, исследованиях нефти и нефтепродуктов, биотоплив и др.
\end{abstract}

\section{Введение}

Масс-спектрометры, пожалуй, наилучшие инструменты, доступные в наше время химику-аналитику. Принцип их действия основан на ионизации исследуемых образцов,разделении и детектировании ионов с различными значениями отношения массы к заряду $(m / z)$ с фемтограммовой (фг) и нанограммовой (нг) чувствительностью, что позволяет расшифровывать химический состав сложных смесей. Неудивительно, что эти приборы нашли широкое применение во многих областях науки и отраслях промышленности. Масс-спектрометр условно можно разделить на три основных блока: ионный источник, масс-анализатор (МА) и детектор. Хотя функции ионного источника и детектора чрезвычайно важны для работы масс-спектрометра, именно выбор типа масс-анализатора оказывается решающим для получения правильных достоверных данных. Матрица анализируемых образцов, требуемые уровни пределов обнаружения, необходимость проводить целевой или нецелевой анализ, бюджет - все эти факторы играют важную роль при выборе масс-анализатора. Существуют четыре типа масс-анализаторов: времяпролетный, квадрупольный, МА с ионной ловушкой и магнитно-секторный.

Магнитно-секторный масс-анализатор обычно используется из-за высокого разрешения для определения точных масс. Хотя этот тип массанализаторов достаточно дорог, он превосходно зарекомендовал себя при количественном определении таких химических загрязнителей, как диоксины. Масс-анализатор с ионной ловушкой 
особенно полезен в тандемной масс-спектрометрии $\left(\mathrm{MC} / \mathrm{MC}\right.$ или $\left.\mathrm{MC}^{\mathrm{n}}\right)$. Тандемные $\mathrm{MC}$ дают информацию о ионах-продуктах, которая может быть использована для определения содержания заданного ограниченного перечня целевых аналитов, подтверждения их химического состава при стандартной процедуре контроля качества. Квадрупольный масс-анализатор - самый простой по конструкции, он недорог, вполне подходит для рутинного анализа. Благодаря небольшому размеру (производится в настольной модификации) и относительной простоте использования, он весьма популярен и может быть установлен в большинстве аналитических лабораторий. Времяпролетный масс-анализатор (ВПМА) способен быстро регистрировать полные масс-спектры во всем диапазоне масс. Отличительная особенность конструкции ВПМА состоит в том, что он практически не подвержен загрязнению. Это невероятное преимущество для любой лаборатории, работающей со сложными образцами различного происхождения (например, при исследовании объектов окружающей среды, продуктов питания, растительных и животных тканей, биологических жидкостей, фракций нефти и нефтепродуктов, биотоплив и др.). Времяпролетные масс-спектрометры выпускают в настольной и напольной модификации, есть модели номинального, субноминального и высокого разрешения, они могут комбинироваться с другими масс-анализаторами в тандемной массспектрометрии (MC/MC).

ГХ-ВПМС обладают способностью регистрировать массспектры с высочайшей скоростью до 500 полных масс-спектров в секунду в полном рабочем диапазоне масс в линейном динамическом диапазоне до пятого порядка. Оснащение лаборатории ГХ-ВПМС выгодно потому, что позволяет осуществлять нецелевой обзорный анализ, опираясь на встроенные в программное обеспечение масс-спектральные библиотеки с прекрасным совпадением экспериментальных масс-спектров с библиотечными данными благодаря алгоритму деконволюции, и одновременно поддерживает быструю газовую хроматографию с точным количественным определением целевых аналитов.

\section{Обзорный анализ при получении данных в полном диапазоне масс}

Роль масс-анализатора состоит в том, чтобы выбрать специфический ион с конкретным отношением массы к заряду $(m / z)$ и транспортировать его к детектору. ВПМА, в отличие от медленных сканирующих МА, способен одновременно и быстро разделять ионы по соотношению массы к заряду в полном диапазоне масс. В ВПМА ионы ускоряются из ионного источника выталкивающим напряжением и фокусируются во времяпролетной трубе на рефлектроне, откуда перенаправляются к детектору (рис. 1). Это основное отличие от квадрупольного масс-анализатора, который, в сущности, является масс-фильтром [1]. В нем используется комбинация постоянного (U) и радиочастотного (V) напряжений, которые подаются попарно в противоположной полярности на четыре параллельных симметрично расположенных электрода круглого сечения. Под действием небольшого ускоряющего напряжения (10-20 В) ионы влетают в МА параллельно осям стержней и под действием задаваемого электродами осцилирующего поля начинают колебаться. Ионы со строго определенными соотношениями массы к заряду $\mathrm{m} / \mathrm{z}$, чьи амплитуды достигают заданных значений, направляются к детектору. Таким образом, масс-спектр регистрируется путем изменения соотношения постоянного и радиочастотного напряжений. Чтобы перекрыть весь диапазон масс, приходится менять

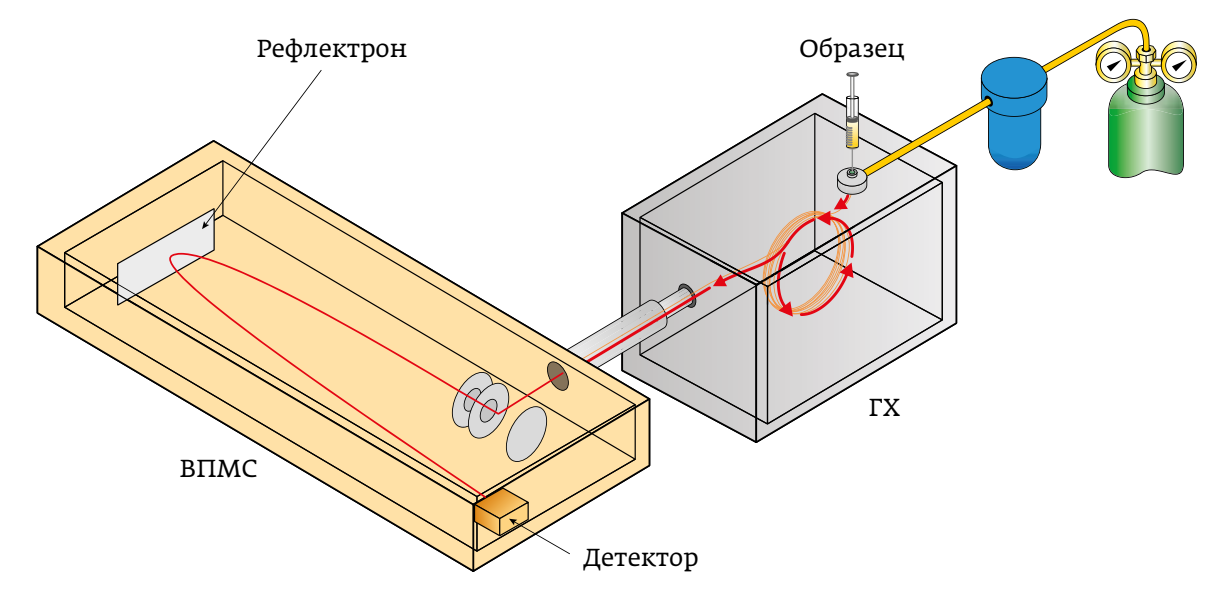

Puc. 1. В системе LECO Pegasus GC-TOFMS, сочетающей газовую хроматографию с времяпролетным масс-спектрометрическим детектированием (ГХ-ВПМС), рефлектрон позволяет различать проникновение ионов в зависимости от их кинетической энергии и отражает их обратно, в направлении к детектору. Это удлиняет траекторию пролета, увеличивает разрешение по массам и улучшает чувствительность, поскольку все ионы с одинаковым m/z достигают детектора одновременно 
отношение $\mathrm{U} / \mathrm{V}$, чтобы все ионы с разными величинами $m / z$ по очереди могли попасть в детектор. Это замедляет скорость регистрации и снижает чувствительность.

Способность ВПМА работать в полном диапазоне масс без ущерба для скорости сбора данных или чувствительности идеально подходит для работы с образцами неизвестного состава. Использование нецелевого анализа полезно для решения многих научно-исследовательских задач, например для поиска потенциальных биомаркеров в метаболомике, обнаружения новых или запрещенных химических веществ в объектах окружающей среды или пищевых продуктах, сбора ценной информации для судебномедицинских экспертиз или в токсикологических исследованиях. Полученная информация может в дальнейшем пригодиться для ретроспективного анализа данных без проведения повторного эксперимента [2].

Метод целевого анализа также выигрывает от получения данных в полном диапазоне масс. В квадрупольном масс-спектрометре регистрация в режиме мониторинга выбранных ионов (SIM) используется для повышения чувствительности по целевым аналитам. В этом режиме ионы с определенным соотношением $\mathrm{m} / \mathrm{z}$ выбираются для контроля содержания целевых аналитов, а остальные ионы в детектор не попадают. Это улучшает пределы детектирования для целевого аналита благодаря увеличению отношения сигнал/шум. Однако полностью теряется массспектральная информация и есть вероятность получения неверных данных, так как матрица может содержать такой же характерный ион и искажать полученные результаты. Собранная об образце информация ограничена только характеристическими ионами с выбранными соотношениями $\mathrm{m} / \mathrm{z}$, которые попадают в детектор. Если потребуются другие дан ${ }^{-}$ ные о пробе или добавление дополнительных аналитов в целевой список, то образец придется снова подготовить и проанализировать повторно. Это не всегда возможно из-за ограничений во времени или доступа к образцам. А при работе в полном диапазоне масс в случае ГХ-ВПМС для целевого количественного анализа тоже можно использовать ионные хроматограммы по характеристическим выделен ${ }^{-}$ ным ионам. Кроме того, в любой момент доступна хроматограмма по полному ионному току и массспектр во всем рабочем диапазоне масс. Если какие-то новые аналиты будут добавлены в список для целевого анализа, повторное проведение эксперимента не потребуется, так как вся информация уже собрана в файле и поиск нового вещества возможен по уже

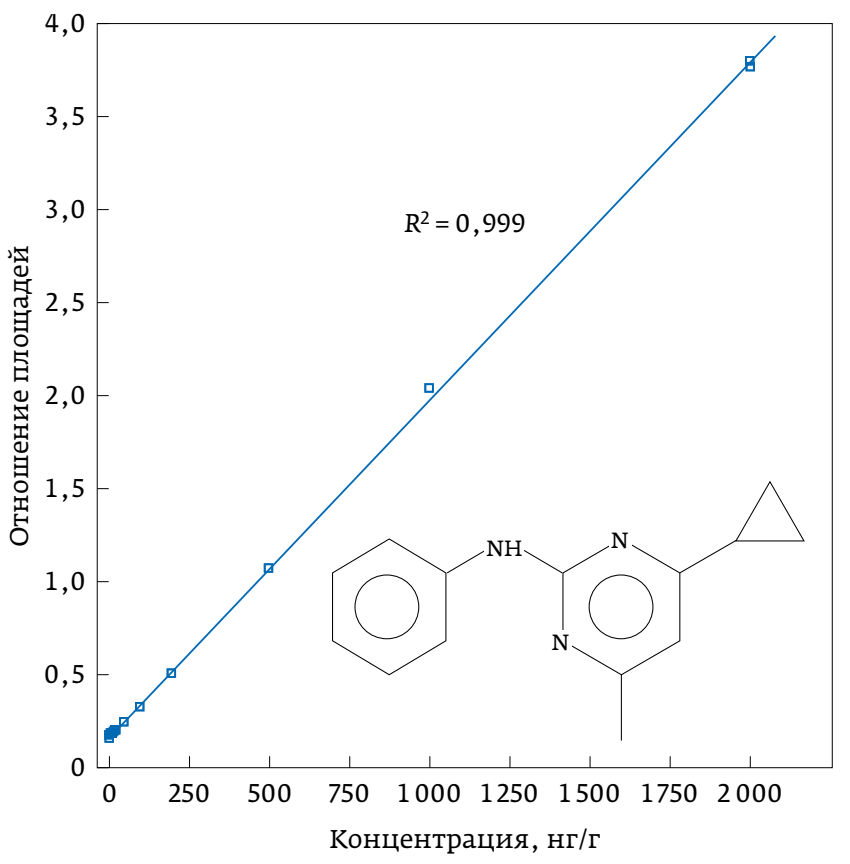

0,1 пг в матрице
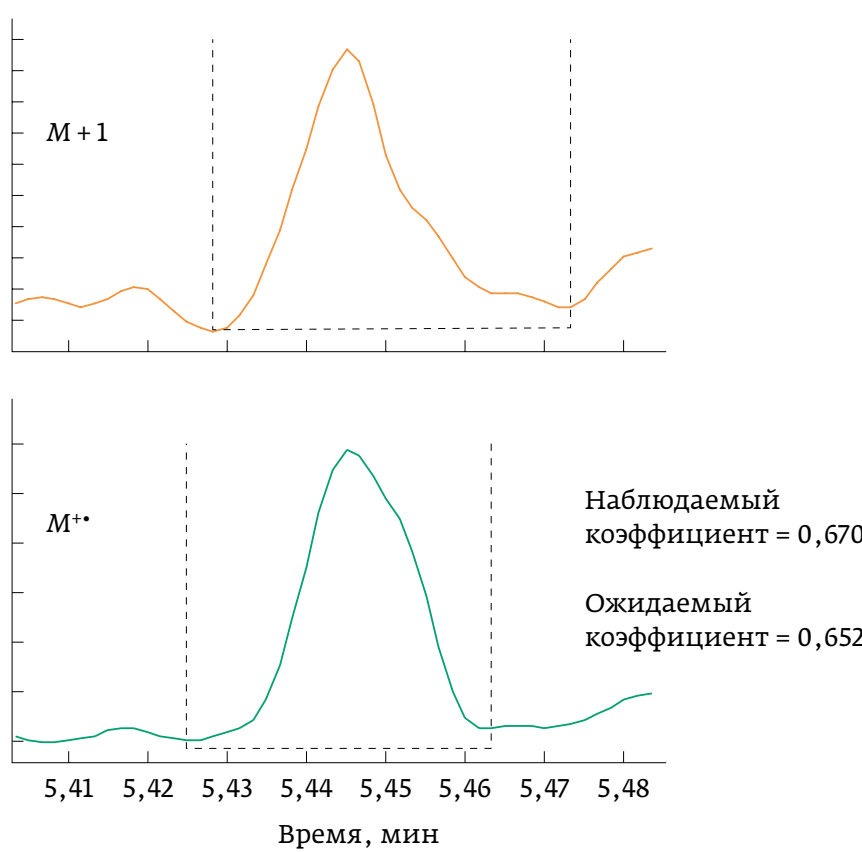

Puc. 2. Калибровочная кривая (от 0,1 до 2000 нг/2) фунгицида ципродинила в матрице клубники. Чувствительность LECO Pegasus ВТ ГХ-ВПМС достигает количественного определения ципродинила ниже контролируемой предельной допустимой концентрации (ПДК). Калибровочная кривая в пищевой матрице также подчеркивает широкий линейный динамический диапазон ВПМС-приборов 
имеющимся аналитическим данным. Кроме того, ВПМС нового поколения откалиброваны таким образом, что позволяют получать более низкие пределы детектирования без потери информации при регистрации данных в полном диапазоне масс (рис. 2).

\section{Лучшая сходимость масс-спектров с библиотечными благодаря}

\section{алгоритму деконволюции}

Использование масс-спектральных библиотек чрезвычайно полезно для ускорения идентификации пиков в хроматограммах. Возможность автоматически сличать качественный эталонный библиотечный масс-спектр с экспериментальным позволяет количественно оценивать коэффициент сходимости. Чем лучше совпадение с данными из библиотеки, тем выше вероятность правильной идентификации. При сканировании в масс-анализаторе, подобном квадрупольному, содержание $\mathrm{m} / \mathrm{z}$-ионов может изменяться в пределах пика даже для одного и того же аналита. Во время сканирования аналит продолжает элюироваться, следовательно, концентрация в источнике изменяется, а вместе с ней и соотношение ионов, поэтому масс-спектр становится несимметричным. Для исключения искажения результаты многократных сканирований должны быть просуммированы и усреднены. В ВПМА нет искажения масс-спектров на разных склонах хроматографических пиков, поскольку ионные пакеты формируются и детектируются одновременно. Поэтому масс-спектры, полученные при помощи ВПМС, оказываются абсолютно идентичными в хроматографическом пике в отличие от данных, получаемых с помощью сканирующего масс-анализатора (рис. 3).

Получение идентичных масс-спектров в разных точках хроматографического пика важно для корректной работы алгоритмов программного обеспечения по автоматической обработке полученных данных (деконволюции, выделения чистых масс-спектров,
Сканирующий MC
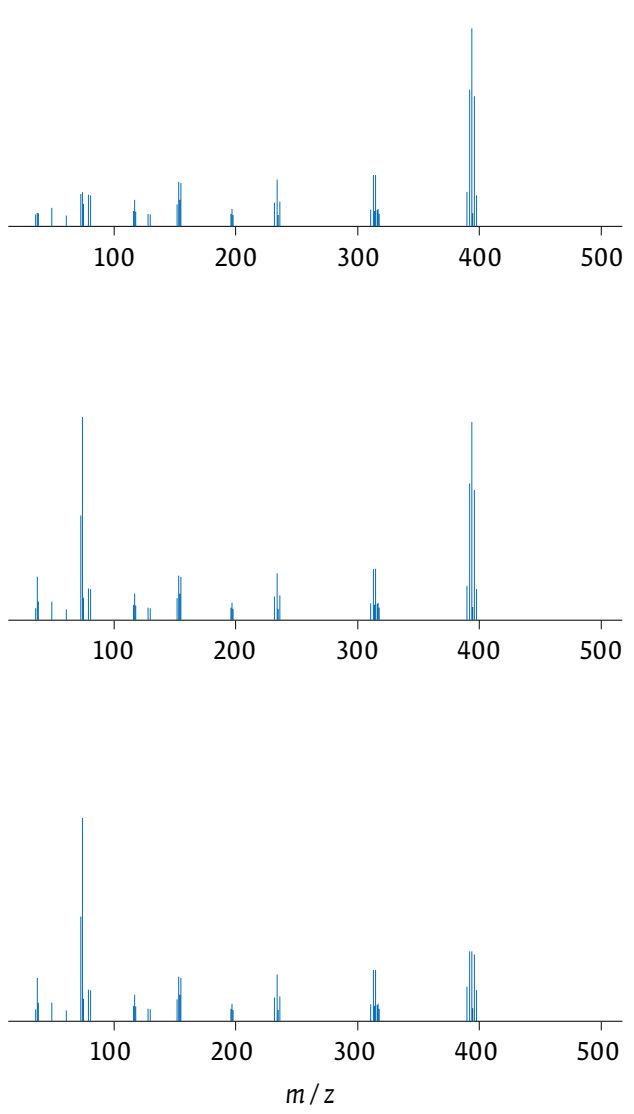

ГХ-пик
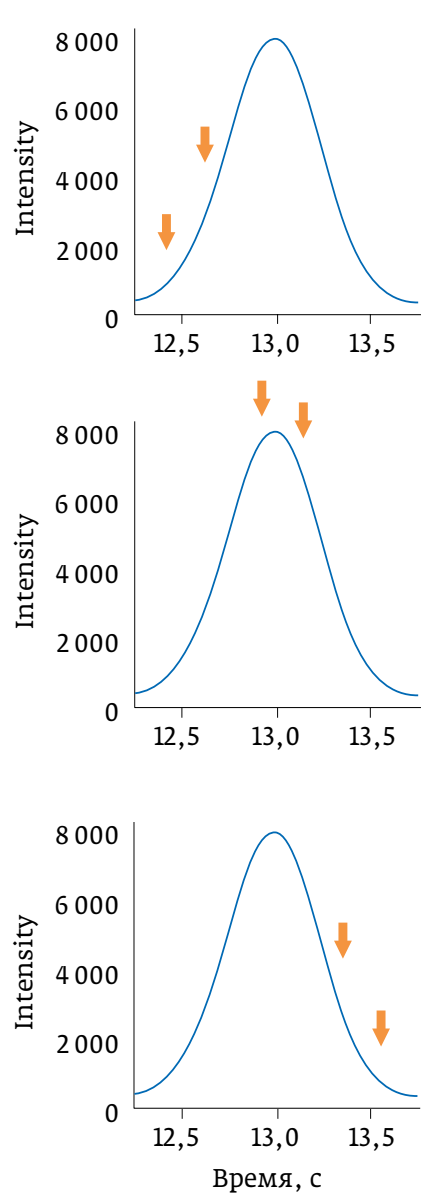

Времяпролетный $\mathrm{MC}$
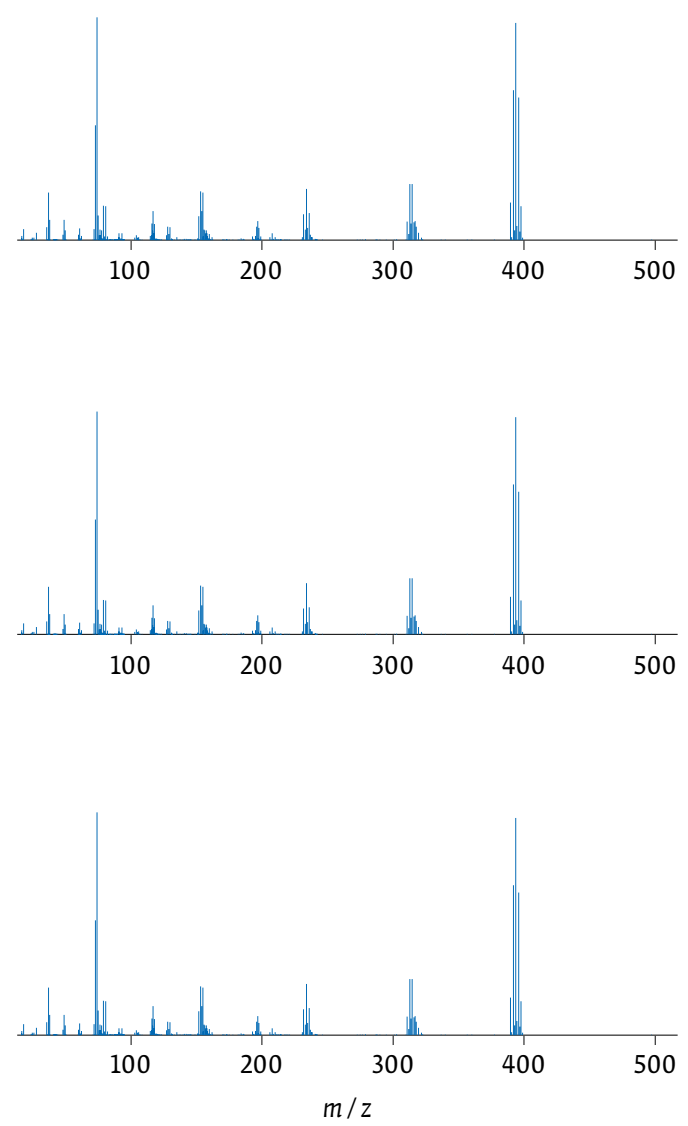

Puc. 3. Отсутствие искажения масс-спектров во ВПМС в сравнении с МС сканирующего типа в разных точках хроматографического пика 

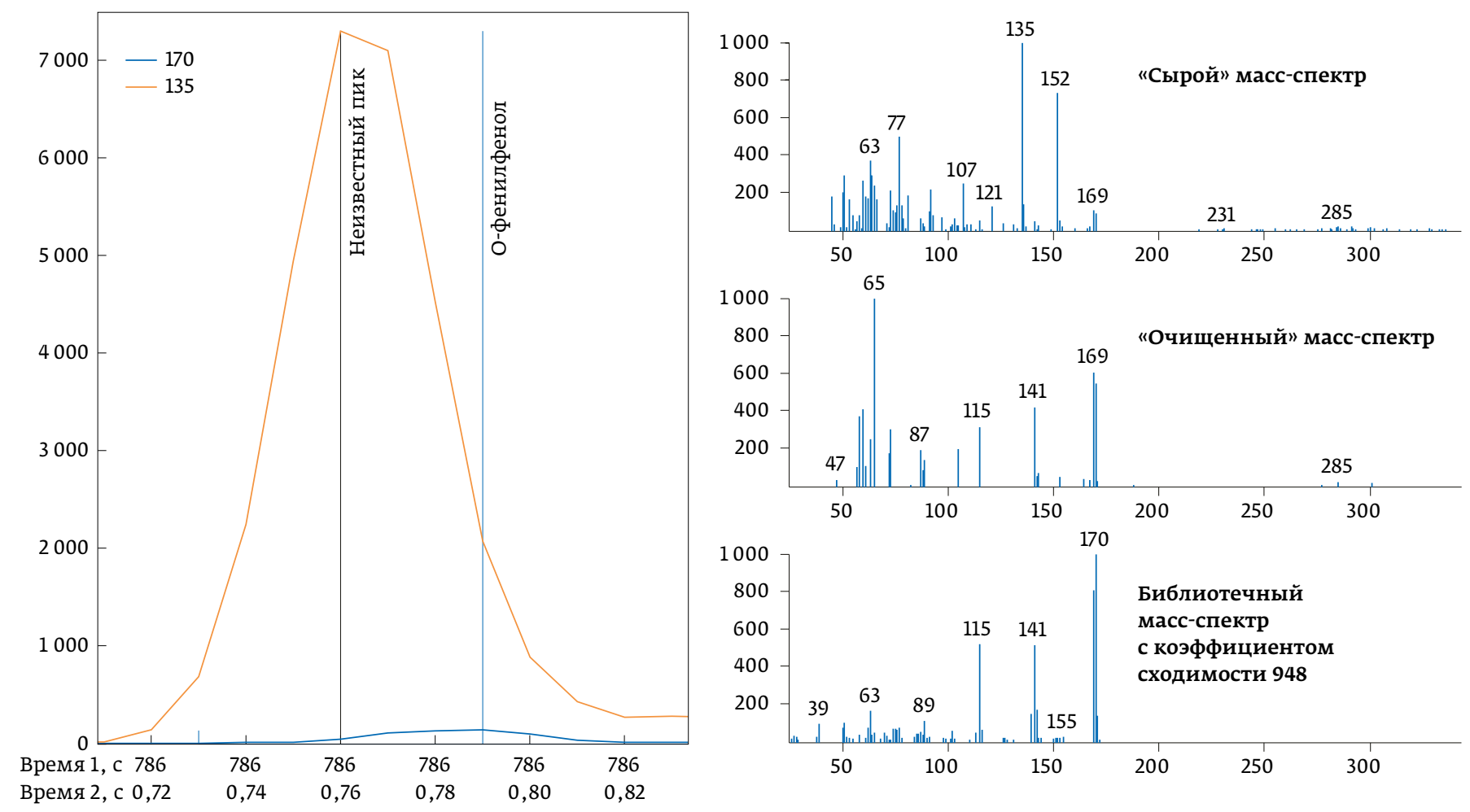

Puc. 4. Анализ пестицидов в травяном чае. Высокое совпадение с библиотечным масс-спектром (948) для о-фенилфенола благодаря масс-спектральной деконволющии. Сырой масс-спектр без деконволющии демонстрирует сильную интерференцию с масс-спектром неизвестного коэлюирующегося компонента матрицы с характеристичным ионом с m / z 135. С помощью ПО ChromaTOF (LECO) со встроенными алгоритмами деконволюции мешающие пики коэлюирующегося компонента быпи удалены

автоматического поиска пиков, сравнения с библиотечными массспектрами, разделения сигналов коэлюирующихся компонентов). Алгоритмы масс-спектральной деконволюции извлекают массспектры аналита из перекрывающихся аналитических сигналов. В результате получается "чистый" масс-спектр, который показывает лучшую сходимость с библиотечным, чем при использовании метода вычитания фона. При этом удается получать высокие коэффициенты сходимости массспектров с библиотечными даже при работе со сложными образцами в экологических, метаболомных, нефтехимических исследованиях. Компоненты матрицы с высоким содержанием в образце могут маскировать хроматографические пики важных минорных

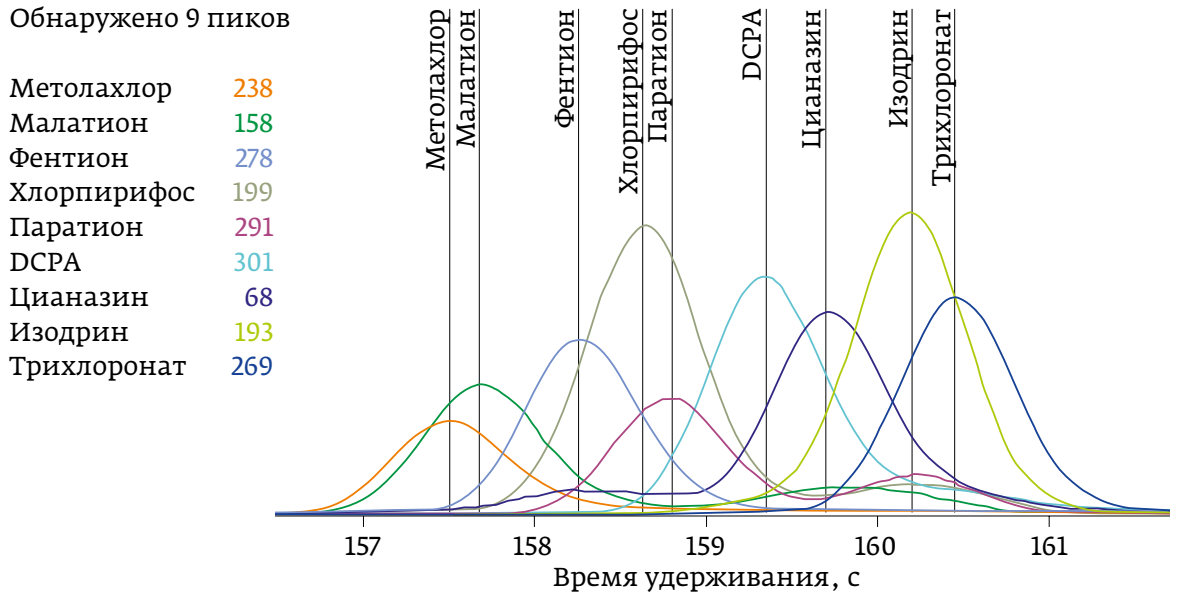

Puc. 5. Масс-спектральная деконволюция и сопоставление данных с библиотечными масс-спектрами позволяет программным алгоритмам находить пики автоматически. Функция автоматического поиска пиков в ПО ChromaTOF сокращает время идентификации аналитов как дяя целевого, так и для нецелевого анализа. Увеличение скорости регистрации до 40 массспектров в секунду с использованием алгоритма деконволюции позволяет идентифицировать девять пестицидов на небольшом участке хроматограммы с временами удерживания в диапазоне от 157 до 161 с 
аналитов, при этом искажая и их масс-спектры. Алгоритмы деконволюции помогают извлекать чистые масс-спектры целевых аналитов с хорошей сходимостью с библиотечными (рис. 4).

Корректность работы алгоритмов деконволюции также зависит от плотности сбора данных, то есть скорости регистрации масс-спектров в MC-детекторе. Небходимо получить достаточное количество точек на пик, чтобы правильно определить положение вершины и геометрию каждого пика. В сложных многокомпонентных образцах, где возможно наложение аналитических сигналов, высокая скорость сбора данных в ВПМС соответствует требованиям осуществления наилучшей масс-спектральной деконволюции (рис. 5).

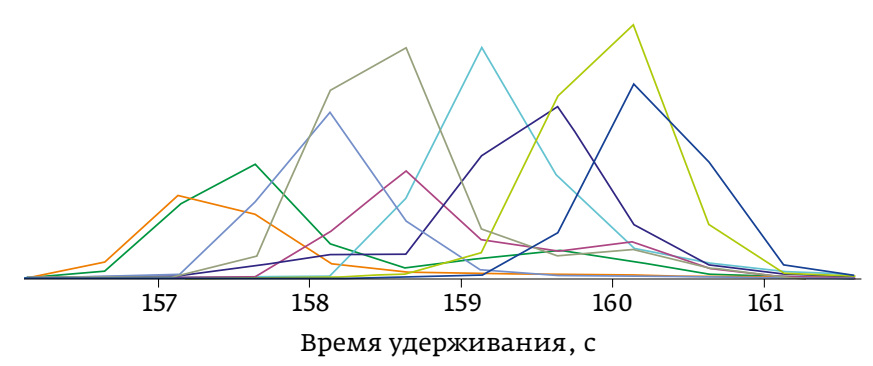

а) Скорость регистрации масс-спектра $=2(1 / \mathrm{c})-4$ точки $/$ пик Автоматизированное обнаружение пика невозможно

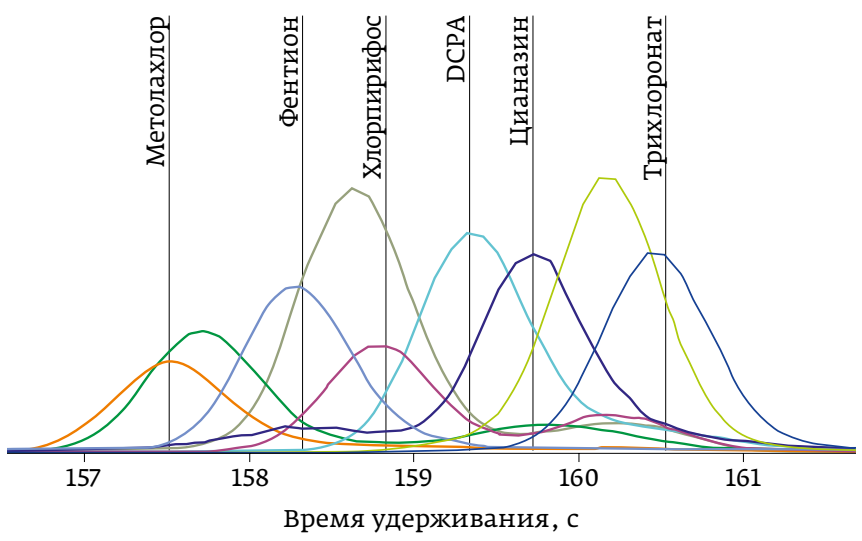

в) Скорость регистрации масс-спектра $=10(1 / \mathrm{c})-20$ точек / пик Обнаружено 6 пиков

\section{Быстрая хроматография \\ с точной количественной оценкой}

Скорость сбора данных важна не только для массспектральной деконволюции, но и для количественного анализа. Для точного описания геометрии пика данные должны быть получены с минимальным разрешением не менее 10 точек на ширину базового разрешенного пика. Этот минимум увеличивают до 15-25 точек для более сложных случаев, когда происходит коэлюирование. Если не собрать достаточное количество данных для описания хроматографического пика, можно пропустить его вершину, что приведет к изменению времени удерживания, следовательно, и индексов, которые также представляют

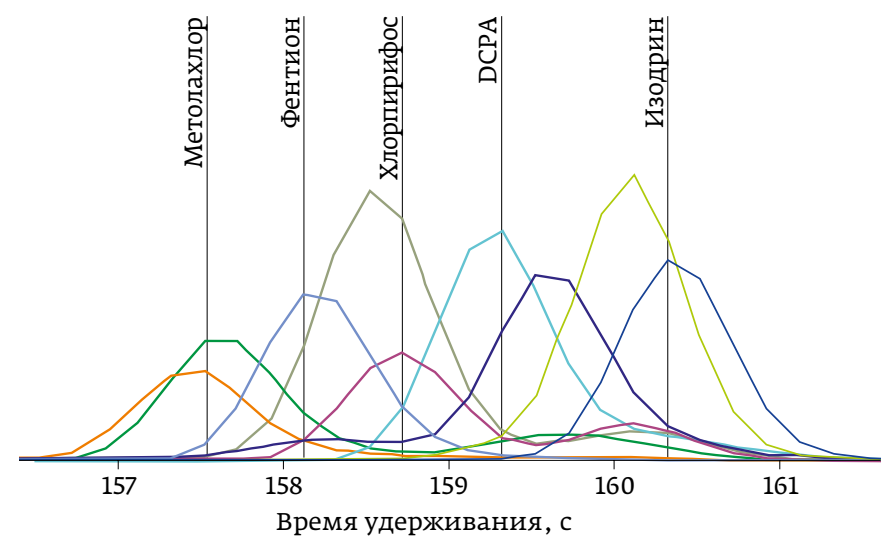

б) Скорость регистрации масс-спектра $=5(1 / \mathrm{c})-10$ точек $/$ пик Обнаружено 5 пиков

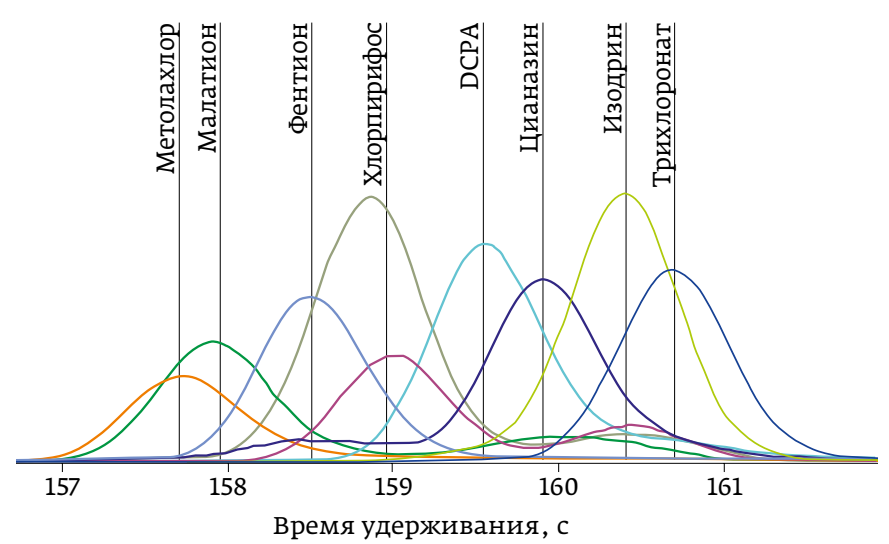

г) Скорость регистрации масс-спектра = 20 (1/c) - 40 точек / пик обнаружено 8 пиков

Puc. 6. Быстрый ГХ-анализ смеси пестицидов приводит к образованию хроматографических пиков шириной 2 с: $а$ - при низкой скорости регистрации масс-спектров (2 $\left.c^{-1}\right)$ автоматический поиск пиков оказывается невозможен; 6 - при увеличении скорости сбора данных до $5 c^{-1}$ достигается плотность данных в 10 точек/ пик, но только 5 из 9 компонентов

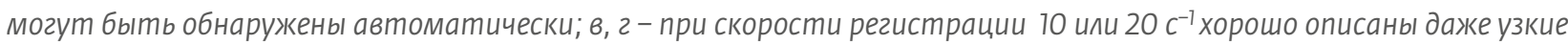
неразрешенные хроматографические пики, поэтому алгоритмы автоматического поиска пиков, встроенные в программное обеспечение ChromaTOF, позволяют их обнаружить и расшифровать по алгоритмам масс-спектральной деконволюции 
важную информацию для идентификации. Кроме того, если пик не описан должным количеством точек, ухудшается воспроизводимость определения его площади, что приводит к увеличению относительных стандартных отклонений в количественном анализе.

Быстрая газовая хроматография реализуется при использовании специальных комбинаций микроколонок, различных газов-носителей, регулировки потоков газа-носителя и программируемых термостатов с быстрыми режимами нагрева. Высокой производительности лаборатории можно добиться за счет сокращения времени анализа. В варианте быстрой ГХ требования к скорости сбора данных МС-детектором возрастают, поскольку пики сужаются и часто происходит коэлюирование компонентов. Чтобы увеличить пропускную способность при массовой выборке без ущерба для качества получаемых результатов, скорости сбора данных должны быть адаптированы для точного определения более узких пиков за более короткий интервал времени. При быстрой хроматографии пики могут иметь ширину 0,5-2 с (рис. 6).

ГХ-ВПМС оказывается полезен в рутинных высокопроизводительных лабораториях, в которых хотят использовать быструю газовую хроматографию. Обычные и чувствительные ко времени данные могут быть точно количественно определены с помощью быстрого ВПМС. Кроме того, высокие скорости сбора данных требуются для реализации принципа полной двумерной газовой хроматографии (2D-ГX), которая незаменима для анализа сложных многокомпонентных образцов. Пики, полученные в результате 2D-ГХ анализа, имеют ширину 50-200 мс, что требует от MC-детектора скорости сбора данных не менее $200 \mathrm{c}^{-1}$ (табл. 1). Скорость же квадрупольного масс-спектрометра ограничена и зависит от выбираемого диапазона масс, при этом ухудшается качество полученных данных и точность анализа.

\section{Выводы}

Хромато-масс-спектрометр - отличный инструмент в любой аналитической лаборатории, а выбор подходящего масс-анализатора может улучшить качество получаемых данных, расширить возможности лаборатории и при этом соответствовать ее бюджету. Времяпролетный хромато-масс-спектрометр - отличный выбор для определения химического состава сложных образцов, обзорного анализа, быстрой или полной двумерной газовой хроматографии (2D-ГX). Времяпролетный хромато-масс-спектрометр может с успехом использоваться в исследовательских
Табиица 1. Скорость сбора данных, необходимая

дия достижения нужной ппотности точек

(10 или 25 точек/пик) и корректного анализа

\begin{tabular}{|c|c|c|}
\hline $\begin{array}{c}\text { Ширина пиков, } \\
\text { с }\end{array}$ & $\begin{array}{c}\text { Скорость сбора данных, } \\
\text { c }^{-1}\end{array}$ & Точки/пик \\
\hline 20 & 0,5 & 10 \\
\hline 20 & 1,25 & 25 \\
\hline 10 & 1 & 10 \\
\hline 10 & 2,5 & 25 \\
\hline 5 & 2 & 10 \\
\hline 5 & 5 & 25 \\
\hline 2,5 & 4 & 10 \\
\hline 2,5 & 10 & 25 \\
\hline 1 & 10 & 10 \\
\hline 1 & 25 & 25 \\
\hline 0,5 & 20 & 10 \\
\hline 0,5 & 50 & 25 \\
\hline 0,2 & 50 & 10 \\
\hline 0,2 & 125 & 25 \\
\hline 0,1 & 100 & 10 \\
\hline 0,1 & 250 & 25 \\
\hline
\end{tabular}

Выделенные цветом ячейки соответствуют скоростям сбора дан ${ }^{-}$ ных, которые не могут быть получены с помощью квадрупольных масс-анализаторов (в диапазоне масс 50-550 m/z).

и контрольно-аналитических лабораториях, работающих в области контроля загрязнения окружающей среды, безопасности продуктов питания, в метаболомике, нефтехимии и органической геохимии, с биотопливами и другими объектами растительного происхождения. Регистрация данных в полном диапазоне масс идеально подходит для нецелевого анализа, масс-спектральная деконволюция упрощает сопоставление с данными масс-спектральных библиотек и работу со сложными образцами, а высокая скорость сбора данных делает возможными режимы быстрой ГХ и 2D-ГХ.

Автор выражает благодарность Джеку Кохрану и LECO Corporation за Аюбезно предоставленные изображения, использованные в статье.

\section{Литература}

1. Binkley J., Libardoni M. Comparing the Capabilities of Time-ofFlight and Quadrupole Mass Spectrometers. LCGC Special Issue. 2010. V. 8 , No. 3, pp. 28-33.

2. Gross J. Mass Spectrometry - A Textbook; Springer: Heidelberg, Germany. 2004. pp. 477-479. 


\section{АНАЛИТИЧЕСКОЕ ОБОРУДОВАНИЕ ОТ МИРОВОГО ЛИДЕРА}

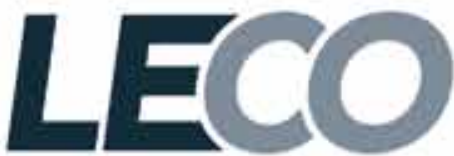

EMPOWERING RESULTS уголь

Быстрое

определение

показателей

качества уәля,

анализ других

органических

материалов

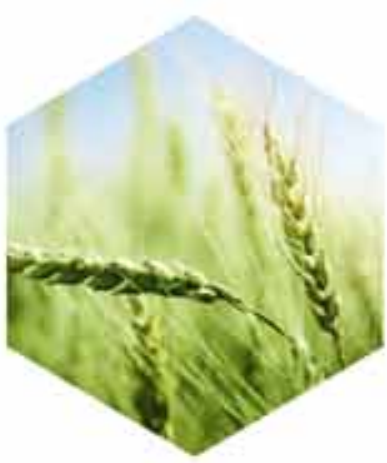

АЗОТ/ПРОТЕИН

Быстрый и нетрудоемкий

анализ удобрений и

продуктов питания

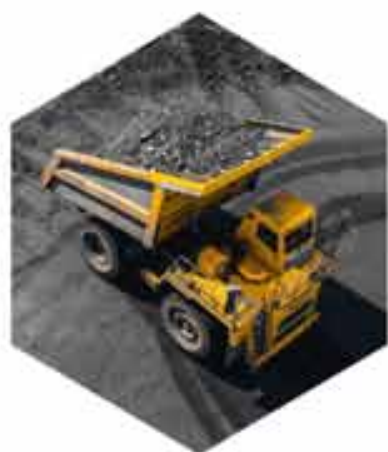

\section{МЕТАЛЛЫ И СПЛАВЫ}

Элементный анализ газообразующих примесей и поспойный

анапиз покрытий

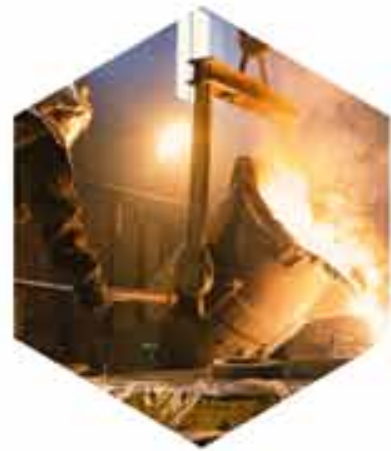

1000

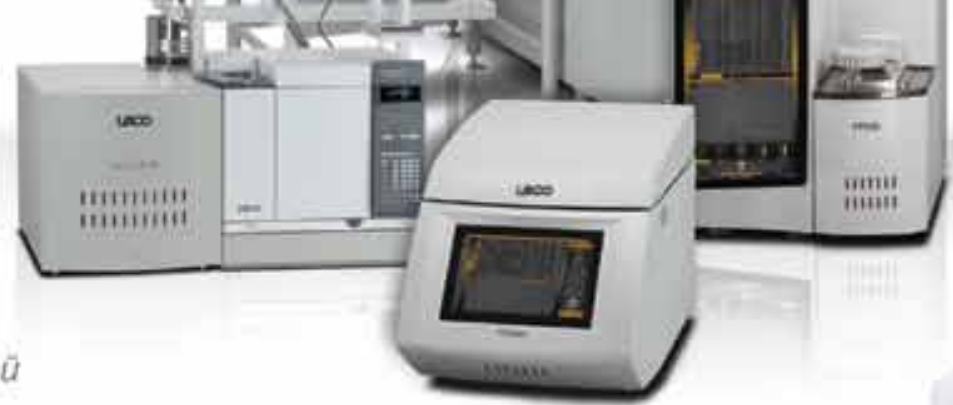

ЭКОЛОГИЯ

Исчерпывающая информация при обзорном анализе в исследованиях

окружающей среды

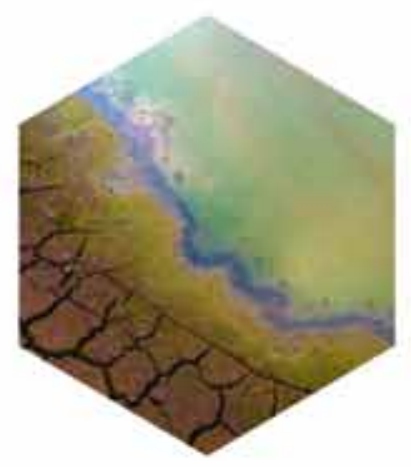

HEФTb

Новые аналитические подходы в нефтедобыче u нестехими

Мы более 80 лет производим оборудование для элементного анализа.

термогравиметрии, хромато-масс-спектрометрии, материалографии.

\section{LЕСО ГАРАНТИРУЕТ ДОСТОВЕРНЫЙ РЕЗУЛЬТАТ!}

